\title{
Relato de Gamificação da disciplina Projeto e Análise de Algoritmos do curso de Engenharia de Computação
}

\author{
Christian Hideki Maekawa ${ }^{1}$, Walter Aoiama Nagai ${ }^{2}$, Claudia Akemi Izeki ${ }^{2}$ \\ ${ }^{1}$ Universidade Federal de Itajubá - Campus Itajubá \\ R. Dr. Pereira Cabral, 1303 - Pinheirinho - 37500-903 - Itajubá - MG \\ christianmaekawa@unifei.edu.br \\ ${ }^{2}$ Universidade Federal de Itajubá - Campus Itabira \\ R. Irmã Ivone Drummond, 200, Distrito Industrial II - 35903-087 - Itabira - MG \\ walternagai@unifei.edu.br, claudiaizeki@unifei.edu.br
}

\begin{abstract}
The capacity and engagement in solve problem is important to a Computer Engineer that should provide solutions for real-world problems in differentiate ways. This capacity and motivation are provided in different methodologies like Gamification. In this article is described a strategy of Gamification of Analysis and Project of Algorithms course that will help to open new approach to motivate the beginner student in the university in collaborate for a better interpretation, engagement in programming and comprehension of various computer algorithms.
\end{abstract}

Resumo. A capacidade de resolução de problemas é uma competência é importante para o egresso de Engenharia da Computação, que deve criar soluções diferenciadas para vários tipos de problemas do mundo real. Essa competência deve ser estimulada por meio de metodologias diferenciadas de transferência de conhecimentos como a Gamificação. Neste trabalho é relatada uma estratégia de Gamificação da disciplina Projeto e Análise de Algoritmos do curso de Engenharia da Computação, permitindo uma nova abordagem para estimular os discentes em colaborar para uma melhor interpretação, facilidade na programação e na compreensão de diversos algoritmos da Computação.

\section{Introdução}

Ministrar aulas expositivas no ensino tradicional enfatizando o poder do professor sobre o estudante, é um relacionamento mestre-aprendiz poderoso quando a transferência de conhecimento é o objetivo primário. Entretanto, quando o objetivo é o pensamento crítico ou a resolução de problemas, tanto professores quanto alunos devem modificar seus papéis e responsabilidades tradicionais. Esse processo requer que os estudantes se tornem ativamente envolvidos em seu próprio aprendizado, realizando suas próprias descobertas do que meramente aceitar falas e escritas.

Uma das várias competências que um ingressante de Engenharia da Computação deve ter é a capacidade de resolução de problemas, que pode ser estimulada com a produção de programas executáveis corretos e com boas práticas de programação. Este desenvolvimento correto de programas é realizado durante os dois primeiros anos do curso de Engenharia da Computação da UNIFEI Campus Itabira nas disciplinas Programação 


\section{CBIE-LACLO 2015}

Anais dos Workshops do IV Congresso Brasileiro de Informática na Educação (CBIE 2015)

Básica, Algoritmos e Estruturas de Dados e Projeto e Análise de Algoritmos, sendo este o objeto de estudo deste artigo.

$\mathrm{Na}$ educação, especificamente nas matérias de programação, segundo [Nagai and Izeki 2013] há o anseio de "possibilitar o desenvolvimento de raciocínio lógico para estabelecer soluções computacionais em um dado problema, empregando técnicas de desenvolvimento de programas corretos e bem estruturados, direcionando estas soluções para codificação". Segundo [Tanaka 2013], "os estudantes que já possuem uma carga de conhecimento sobre a computação tendem a corrigir o seu conhecimento utilizando o aprendizado construtivista". Para [Tanaka 2013], "metodologia de ensino Gamificada se torna mais adaptável às expectativas dos alunos, fornecendo motivação para estudar não apenas para obter sucesso na disciplina e sim para concretizar seu conhecimento".

Em [Huang and Soman 2013] são descritas experiências de Gamificação realizadas na Universidade de Toronto em várias áreas, inclusive ciências sociais e administração. Em conformidade com o tema de Gamificação de disciplinas da área de Computação, no trabalho de [Iosup and Epema 2014] são descritas experiências de Gamificação para a disciplina de Organização de Computadores e a disciplina de pós-graduação Cloud Computing de uma universidade na Europa, na qual frequentaram durante três anos 450 discentes nessas disciplinas. No trabalho de [Kumar and Khurana 2012] é descrita a Gamificação da disciplina de programação introdutória em linguagem $\mathrm{C} / \mathrm{C}++$ para aumentar o engajamento dos discentes, transformando requisitos da disciplina em itens de jogo.

Neste artigo é feito um relato da experiência de Gamificação da disciplina de Projeto e Análise de Algoritmos do curso de Engenharia da Computação da UNIFEI - Campus Itabira. Para este relato, o artigo encontra-se organizado da seguinte forma: na Seção 2 é realizada uma revisão bibliográfica de Gamificação e a descrição da Gamificação aplicada à disciplina; na Seção 3 são descritas algumas avaliações empíricas e os resultados obtidos da Gamificação na disciplina, e por fim, na Seção 4 são apresentadas as considerações finais do artigo.

\section{Modelagem da Gamificação}

A Gamificação é um termo descrito por [Kapp 2012], que consiste em uma ferramenta responsável por auxiliar na solução de desafios e instigar a motivação dos participantes. Essa ferramenta utiliza estratégias baseadas em jogos, que são adaptáveis e aplicáveis em diversas áreas, como: empresas, mercado de trabalho, instituições financeiras, saúde e educação [Vianna et al. 2013].

A Gamificação é composta por elementos que podem ser recombinados de maneiras diferentes para a construção de um jogo. Esses elementos são a dinâmica, mecânica e os componentes do jogo. Segundo [Alves 2014], a dinâmica é constituída por elementos que auxiliam na cognição e experiências reais. A mecânica de um jogo é responsável pelas ações executadas pelo jogador e o andamento do jogo. Por fim, tem-se os componentes do jogo, que são formas especiais que complementam o que a dinâmica e a mecânica representam, ou seja, fazem parte do acabamento. 


\subsection{Projeto e Análise de Algoritmos}

Assim como no trabalho de [Kumar and Khurana 2012], no qual os conceitos de algoritmos e análise são mais difíceis de incorporar no dia a dia dos estudantes de Computação, a disciplina de Projeto e Análise de Algoritmos engloba grande parte do conteúdo visto até então pelo discente de Engenharia de Computação, possibilitando o desenvolvimento de um raciocínio lógico mais aprimorado para estabelecer soluções computacionais eficientes em um dado problema, empregando técnicas de desenvolvimento de programas corretos e bem estruturados. O conteúdo ministrado nessa matéria colaborará para uma melhor interpretação, facilidade na programação e na compreensão no funcionamento de diversos algoritmos.

Enfim, o conteúdo da disciplina Projeto e Análise de Algoritmos aborda os conteúdos ministrados nas disciplinas anteriores, com um enfoque em análises de complexidades dos algoritmos de diferentes técnicas de implementação. A disciplina acontece em uma carga horária de 64 horas em 16 semanas de aula e todas as aulas são ministradas em sala. Outras atividades da disciplina acontecem extraclasse. As notas da disciplina variam de 0 (zero) a 100 (cem) pontos por bimestre, na qual um discente é aprovado com uma média 60 pontos ao término da disciplina. Neste artigo será descrita a experiência realizada com 29 discentes no segundo semestre de 2014.

\subsection{Dinâmica}

O jogo da disciplina de Projeto e Análise de Algoritmos foi denominado Treinamento de Jedi Hacker (TJH), no qual a sua dinâmica se baseia na narrativa (storytelling) da mitologia de ficção científica Star Wars ${ }^{1}$ criada por George Lucas. Essa narrativa acontece após os eventos dos 6 filmes da saga Star Wars, que mostra a preocupação com as informações do Império após a sua queda no sexto filme: Episódio 6 - Retorno do Jedi. Assim, Luke, Leia e Han Solo precisam criar uma categoria de jedis, Jedi Hackers. A narrativa da disciplina situa-se entre o episódio 6 e o vindouro episódio 7 que será divulgado no final do ano de 2015. Com essa narrativa se permite criar um vínculo sentimental e mesmo de superação ao associar os discentes da disciplina com aprendizes, cavaleiros e mestres jedis.

\subsection{Mecânica}

A mecânica do TJH foi baseada nos seguintes elementos:

- desafios: os discentes foram convidados a participar de 8 treinamentos jedis que aconteceram durante os dois bimestres em sala de aula com uma carga horária de 2 horas/aula para cada treinamento. Esses treinamentos possuíam a característica de associar os conhecimentos repassados pelo docente a fim de estimular os discentes a resolver um ou mais problemas por treinamento;

- cooperação: durante o jogo, os discentes em equipe podiam desenvolver projetos práticos em linguagem $\mathrm{C}++$ ou Java que possuíam o mesmo contexto da narrativa;

- feedback: o feedback do jogo era realizado pelas respostas dos exercícios teóricos, treinamentos jedi e avaliações em sala de aula que eram providas pelo docente;

- recompensas: com o progresso obtido pelos docentes, existia uma pontuação acumulada que permitia que os discentes subissem de níveis de escala jedi. Esses níveis serão descritos na próxima subseção;

\footnotetext{
${ }^{1}$ Mais informações podem ser obtidas em: http: //www. starwars . com.
} 
- transações: pelas recompensas obtidas alguns tipos de troca podiam ser realizadas. A partir dos níveis Cavaleiro e Mestre, que eram os últimos, algumas recompensas podiam ser usadas pelos discentes, tais como: pulo, telecinese, velocidade e truque mental. O pulo permitia eliminar uma questão em qualquer avaliação escrita, adiar um dia a entrega de um projeto ou revisar uma avaliação após a data combinada com o docente. A telecinese permitia adicionar 10 pontos em uma avaliação por bimestre. A velocidade permitia ao discente um trabalho adicional para aumentar a média final. A habilidade de truque mental permite alterar a nota de um projeto prático para 60 pontos.

\subsection{Componentes}

Os componentes do jogo TJH foram:

- realizações: todos os discentes eram gratificados por pontos de avaliações escritas, projetos práticos, listas de exercícios realizadas, treinamentos, interações sociais e participação em monitoria;

- badges: a cada nível jedi obtido pelo discente, o mesmo era gratificado com uma medalha que representava o nível recém-adquirido;

- placar: os discentes podiam acompanhar as atividades concluídas e a pontuação recebida por meio de e-mails enviados pelo docente;

- níveis: O primeiro nível era Youngling, o segundo nível era Padawan, terceiro nível era Cavaleiro Jedi, quarto nível era Mestre Jedi e por último o nível Jedi Conselheiro. A cada um era atribuída uma porcentagem concluída no jogo. Um jogador se tornava Youngling tendo-se até 39\% dos pontos, um Padawan era aquele que possuía até $59 \%$ dos pontos, um cavaleiro possuía até $69 \%$, um mestre possui até $89 \%$ dos pontos e o conselheiro possuía até $100 \%$ dos pontos;

- pontos: O jogo possuía uma escala de pontos que varia de 0 (zero) à 6020 (seis mil e vinte pontos).

\subsection{Pontuações}

As pontuações do jogo aconteciam de acordo com a Tabela 1, que relacionava as atividades da disciplina com itens do jogo. A tabela foi dividida em atividade, a denominação jedi no TJH e as pontuações máximas de cada atividade. As atividades ocorriam em 2 bimestres distintos. Além das atividades da disciplina que pontuavam 5800 pontos, também eram contabilizados pontos extras de presença em monitoria e interações com o docente, que eram distribuídos 200 e 20 pontos, respectivamente. Ou seja, o jogador/discente poderia ter no máximo 6020 pontos durante todo o jogo TJH.

\section{Avaliação Empírica}

Com as pontuações atribuídas para cada discente durante a realização da disciplina foram produzidos alguns gráficos de desempenho dos estudantes do segundo semestre de 2014 da disciplina. Na Figura 1 são apresentadas as pontuações finais dos discentes no final do semestre. Ao analisar a Figura 1, pode-se observar que os discentes 7 e 9 e que dois discentes 10 e 19 pontuaram menos de 1000 pontos. A razão para isso é que os discentes 7 e 9 não frequentaram a disciplina; e os discentes 10 e 19 desistiram durante o semestre. Os demais variaram até no máximo de 5000 pontos, que caracterizaria $83 \%$ da pontuação máxima, ou seja, nenhum dos discentes se tornou um Jedi Conselheiro. 


\begin{tabular}{|c|c|c|}
\hline \multicolumn{3}{|c|}{ Primeiro bimestre } \\
\hline Atividade & Denominação no jogo TJH & Pontuação \\
\hline Lista de exercícios \#1 & Lista Podracer & 250 \\
\hline Lista de exercícios \#2 & Lista A-Wing & 250 \\
\hline Projeto \#1 & Projeto R2D2 & 400 \\
\hline Projeto \#2 & Projeto Luke & 600 \\
\hline 4 Exercícios em sala & 4 Treinamentos Jedi & 400 \\
\hline Avaliação parcial \#1 & Teste \#1 & 400 \\
\hline Avaliação parcial \#2 & Teste \#2 & 600 \\
\hline \multicolumn{2}{|c|}{ Total de pontos } & 2900 \\
\hline \multicolumn{3}{|c|}{ Segundo bimestre } \\
\hline Atividade & Denominação no jogo TJH & Pontuação \\
\hline Lista de exercícios \#3 & Lista X-Wing & 250 \\
\hline Lista de exercícios \#4 & Lista Y-Wing & 250 \\
\hline Projeto \#3 & Projeto C3PO & 400 \\
\hline Projeto \#4 & Projeto Yoda & 600 \\
\hline 4 Exercícios em sala & 4 Treinamentos Jedi & 400 \\
\hline Avaliação parcial \#3 & Teste \#3 & 400 \\
\hline Avaliação parcial \#4 & Teste \#4 & 600 \\
\hline \multicolumn{2}{|c|}{$\begin{array}{l}\text { Total de pontos } \\
\end{array}$} & 2900 \\
\hline
\end{tabular}

Tabela 1. Pontuações do jogo distribuídas entre as atividades realizadas na disciplina Projeto e Análise de Algoritmos.

Na Figura 2, pode-se observar o desempenho de todos os discentes nos bimestres. A primeira série determinada por uma linha e losango caracteriza a pontuação máxima de cada discente para o primeiro bimestre. A segunda série determinada por uma linha e quadrado caracteriza a pontuação obtida pelo discente no segundo bimestre. O uso de gráficos por bimestre tem o objetivo de mostrar o desempenho do jogador/discente para perceber ou identificar variações dos pontos como queda ou aumento durante o semestre na disciplina.

Na Figura 3, pode-se observar o desempenho nos dois bimestres pelos alunos que obtiveram as 10 maiores pontuações do jogo, ou seja, o primeiro colocado é o discente com maior pontuação final e o segundo colocado é o discente com a segunda maior pontuação final e assim por diante. A série com linha e losango é relacionada com o primeiro bimestre, a série com linha e quadrado é relacionada com o segundo bimestre. Pelo gráfico da Figura 3, pode-se perceber que os discentes na posição 3, 4 e 9 tiveram um desempenho melhor no segundo bimestre, enquanto os discentes 5, 6, 7 tiveram uma queda durante o jogo.

\subsection{Impacto da Gamificação}

Foi realizada uma avaliação do impacto da Gamificação por meio de um formulário impresso passado aos estudantes contendo algumas perguntas relacionadas a preferências e opiniões da condução da disciplina por meio de Gamificação. Algumas perguntas ainda estão em análise, mas alguns testemunhos podem ser destacados:

- Muito bom o jogo e os treinamentos, aprendemos muito mais fazendo 
CBIE-LACLO 2015

Anais dos Workshops do IV Congresso Brasileiro de Informática na Educação (CBIE 2015)

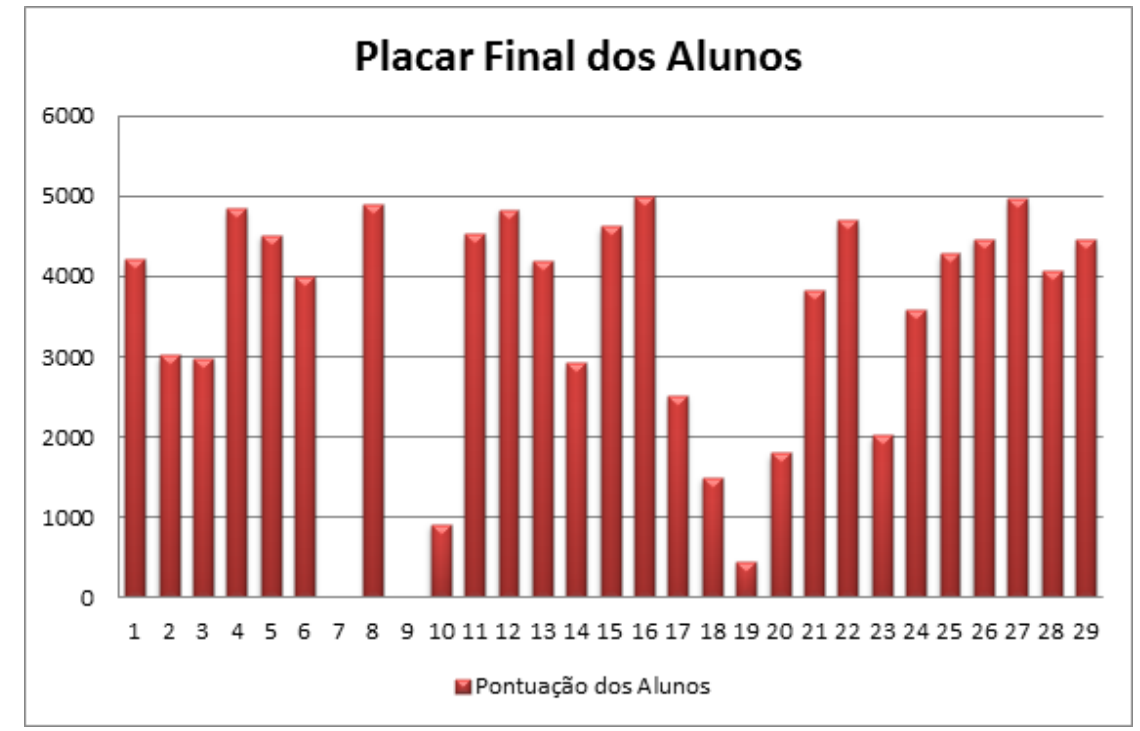

Figura 1. Pontuação final de cada aluno da disciplina.

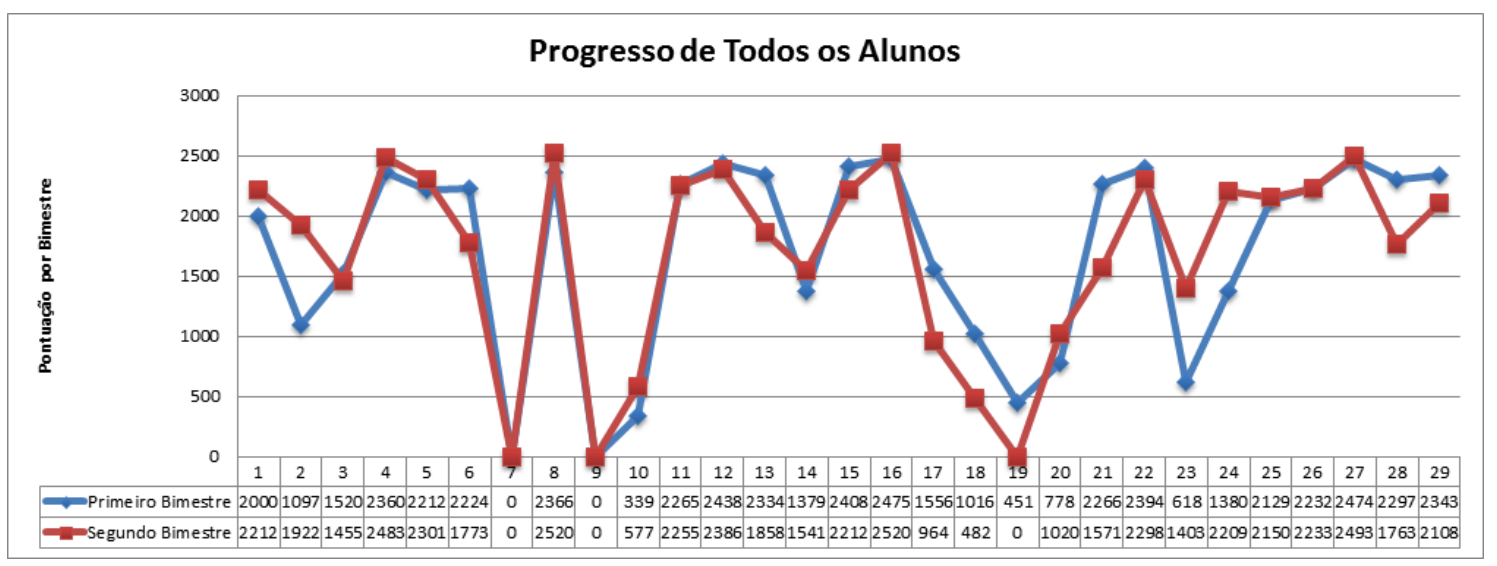

Figura 2. Desempenho de todos os alunos nos dois bimestres

treinamentos do que assistindo aulas faladas em geral. O método de ensino está ótimo.

- Foi uma metodologia muito interessante, estimulando o aluno frequentemente.

- Didática interessante e diferente.

- Bacana a ideia. Podia variar o tema a cada semestre.

- O Tema deverá continuar, mas a distribuição dos pontos poderia ser modificada.

- Promover maior competitividade entre os jogadores do rank.

- Melhorar um pouco a pontuação dos testezinhos (melhorar recompensas).

- Acho que seja opinião pessoal, mas penso que os enunciados dos treinamentos foram um pouco vagos.

- Listas e Trabalhos não valeram pontos de verdade na disciplina, me desmotivou a fazê-los. Pela pontuação no jogo, ficava meio dificil de conseguir os pontos na disciplina.

- Não participei ativamente do jogo, mas considero que faltam maneiras de se premiar discentes que façam coisas inovadoras ou que cumpriram desafios além dos demais. o jogo foi linear 
CBIE-LACLO 2015

Anais dos Workshops do IV Congresso Brasileiro de Informática na Educação (CBIE 2015)

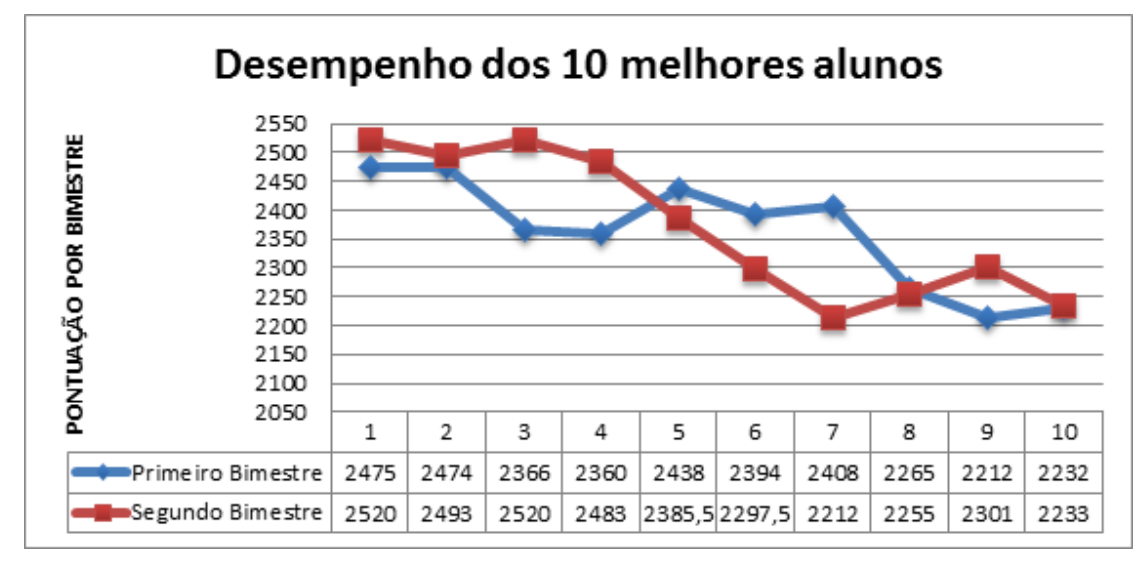

Figura 3. Desempenho dos 10 melhores alunos nos dois bimestres.

demais.

Além desses testemunhos, os discentes destacaram em ordem de preferência de maior para menor, os projetos, a avaliação escrita, os treinamentos Jedi, listas de exercícios, a interação social e por último o auxílio da monitoria para execução das atividades extraclasse.

\subsection{Custo da Gamificação}

A cada semana da disciplina eram necessários:

- preparação do docente para ministrar o conteúdo semanal;

- preparação dos treinamentos jedi que tinham uma periodicidade variada de acordo com o conteúdo ministrado pelo docente. No mesmo dia, eram divulgadas as respostas dos treinamentos jedi para que os discentes pudessem ler e compreender o conteúdo;

- preparação de exercícios semanais e divulgação das respostas dos exercícios da semana anterior;

- preparação de textos dos projetos práticos a cada mês com níveis de dificuldades diferenciados. O primeiro e o terceiro meses tinham uma dificuldade menor de implementação que os projetos do segundo e quarto meses;

- divulgação atualizada do placar de pontuação dos discentes no jogo.

\section{Considerações finais}

Segundo [Smith-Robbins 2011], um jogo é identificado tendo-se três características básicas:

1. objetivo, ou seja, cada jogo tem uma condição de vitória;

2. presença de obstáculos, pois jogos fáceis não são muito divertidos de jogar;

3. colaboração ou competição, que são jogos em que ganhar é determinado ao derrotar outro jogador, ou aqueles em que a vitória é determinada por vencer o jogo em si. O primeiro pode criar concorrência entre os jogadores. O segundo incentiva um jogador para competir contra ele mesmo até atingir o objetivo do jogo.

Dentro desse contexto, a narrativa do jogo permitia uma sensação de vitória parcial devido ao último nível conquistado pelos discentes. Muitos se sentiam satisfeitos em se 
tornar um cavaleiro Jedi, mas tinham sempre o objetivo de se tornar um Mestre Jedi. Os obstáculos do jogo, na verdade, eram os próprios componentes do jogo como treinamento Jedi, as avaliações escritas e os projetos. Apesar que o treinamento Jedi eram um grande incentivo dos discentes participarem em dupla ou grupos para resolução de problemas. A colaboração era mais percebida em projetos práticos realizados em equipe.

O desenvolvimento de Gamificação em uma disciplina no curso de Engenharia de Computação tornou-se um desafio para o docente, além do tempo que se deve dispor para realizar a Gamificação. Com a experiência do segundo semestre de 2014, o docente percebeu a necessidade de:

- uma gestão do jogo, preferencialmente, em ambiente computacional que possa ser acessado por computador ou dispositivo móvel, semelhante à plataforma desenvolvida no trabalho de [Swacha and Baszuro 2013];

- explicar melhor a dinâmica e a mecânica do jogo aos discentes;

- oferecer testes complementares com feedback imediato e com uma frequência que os discentes possam participar mantendo o foco e o engajamento;

- oferecer um número maior de medalhas para incentivar os discentes a participar do jogo;

- avaliar o desempenho individual e da colaboração entre os integrantes das equipes dos projetos práticos;

- oferecer lições de casa complementares para obter feedback do jogo ao término de um conteúdo da disciplina.

A disciplina ocorrerá novamente no segundo semestre de 2015 e algumas das necessidades identificadas serão resolvidas. A intenção do docente da disciplina é continuar com a Gamificação, melhorando seus mecanismos de aplicação e tornando a disciplina mais fácil de ser frequentada e administrada, pelo aumento do engajamento dos próprios discentes.

\section{Agradecimentos}

Agradecimentos à Fundação de Pesquisa de Minas Gerais (FAPEMIG) pelo auxílio financeiro e a Universidade Federal de Itajubá pelo espaço, técnicos e docentes que auxiliaram na execução do trabalho.

\section{Referências}

Alves, F. (2014). Gamification: como criar experiências de aprendizagem engajadoras. DVS Editora.

Huang, W. H.-Y. and Soman, D. (2013). A Practitioners Guide to Gamification of Education. University of Toronto, University of Toronto, 105 St. George Street, Toronto, ON M5S 3E6.

Iosup, A. and Epema, D. (2014). An experience report on using gamification in technical higher education. In Proceedings of the 45th ACM Technical Symposium on Computer Science Education, SIGCSE '14, pages 27-32, New York, NY, USA. ACM.

Kapp, K. M. (2012). The gamification of learning and instruction: game-based methods and strategies for training and education. John Wiley \& Sons. 
Anais dos Workshops do IV Congresso Brasileiro de Informática na Educação (CBIE 2015)

Kumar, B. and Khurana, P. (2012). Gamification in education - learn computer programming with fun. International Journal of Computers and Distributed Systems, 2(1):4653.

Nagai, W. A. and Izeki, C. A. (2013). Relato de experiência com metodologia ativa de aprendizagem em uma disciplina de programação básica com ingressantes dos cursos de engenharia da computação, engenharia de controle e automação e engenharia elétrica. RETEC.

Smith-Robbins, S. (2011). "this game sucks": how to improve the gamification of education. New Horizons, pages 58-59.

Swacha, J. and Baszuro, P. (2013). Gamification-based e-learning platform for computer programming education. X World Conference on Computers in Education, pages 122130 .

Tanaka, E. M. (2013). Estudo e desenvolvimento de uma metodologia de ensino gamificada. Technical report, Instituto de Ciências Matemáticas e de Computação - ICMC.

Vianna, Y., Vianna, M., Medina, B., and Tanaka, S. (2013). Gamification, inc. como reinventar empresas a partir de jogos. Internet. 